\title{
Social Media Use as a Tool to Facilitate or Reduce Cyberbullying Perpetration: A Review Focusing on Anonymous and Nonanonymous Social Media Platforms
}

\author{
Christopher P. Barlett, PhD, Caroline C. DeWitt, Brittany Maronna, and Kaleigh Johnson
}

\begin{abstract}
In today's technologically savvy culture, social media has emerged as a primary method of communicating with others all over the world. Although social media use has many positive benefits, some individuals may use social media to harm others, termed cyberbullying. In this review, we discuss how social media can aid in the facilitation of anonymity perceptions to increase the likelihood of cyberbullying perpetration. We note the theoretical underpinnings relating anonymity perceptions to cyberbullying perpetration and delve into how certain social media platforms that are more or less anonymous (from the user's perspective) can influence cyberbullying. We conclude with discussing how social media can be a tool to help reduce cyberbullying perpetration through decreasing anonymity perceptions of users. Our overall objective is to highlight the aspects of social media use that apply to theoretical postulates to predict cyberbullying to better understand the situations under which individuals use social media to harm others.
\end{abstract}

Keywords: social media, cyberbullying, anonymity

\section{Introduction}

B RANDY VELA (1998-2016) SHOT HERSELF in the chest in front of her family in her living room after being cybervictimized about her appearance. Andres Villagomez and Karinthya Romero were arrested and charged with various crimes after creating fake Facebook accounts to bully Brandy online (Ferguson 2017).

Amanda Todd (1996-2012) hanged herself in her home after being blackmailed and then bullied online regarding an online picture of her showing her breasts. The perpetrator would circulate the picture using various online formats, including Facebook, causing Amanda's peers to tease her both inperson and online (Nobullying.org 2017).

Megan Meier (1992-2006) hanged herself in her closet after an online acquaintance sent her an online message (using MySpace) indicating that the world would be a better place without her. However, the MySpace account and online acquaintance were both fake, created by a parent of Megan's peer who established the fake account to monitor what Megan was saying about her daughter (Pokin 2007).

Ryan Halligan (1989-2003) hanged himself in the bathroom of his home after being cybervictimized on American
Online (AOL) instant messenger. His peers spread an online rumor that he was gay and a female peer pretended to befriend him online before publicly disavowing his affections at school (PBS 2008).

These cases illustrate how cyberbullying victimization can have deleterious effects on teens and emerging adults - and myriad examples abound. Cyberbullying, defined as repeatedly harming another person or group through electronic or digital means (Englander et al. 2017; Menesini et al. 2012; Tokunaga 2010; Vandebosch and Van Cleemput 2008), is an important societal issue. Indeed, a world-wide survey of youth (aged 817) showed that $37 \%$ of youth reported being cybervictimized and $24 \%$ of the same sample reported cyberbullying others (Microsoft 2012). Although cyberbullying victimization was likely not the only causal factor explaining the suicides in the aforementioned examples, extensive research has shown that cyberbullying victimization is related to a litany of negative psychological outcomes, such as depression, anxiety, decreased self-esteem, loneliness, somatic and emotional problems, and suicide ideation (see Kowalski et al. 2014 for a meta-analysis). Reducing cyberbullying perpetration and, by extension, cyberbullying victimization is paramount. Indeed, one commonality that the four aforementioned suicide tragedies share

Department of Psychology, Gettysburg College, Gettysburg, Pennsylvania. 
is that social media platforms were the primary method by which the cyberbullying perpetration occurred. Our review discusses the role of social media in enhancing and inhibiting cyberbullying perpetration by specifically focusing on anonymity perceptions afforded by social media platforms to the user. It is not our objective to label certain social media platforms as more or less tolerant of cyberbullying practices due to how anonymous the user is perceived to be. Rather, we discuss the theoretical postulates and research linking anonymity perceptions with cyberbullying perpetration to illustrate how social media platforms can facilitate cyberbullying perpetration. We conclude with recommendations for how social media platforms can aid in the reduction of cyberbullying by creating a less anonymous online environment. Overall, we hope that the research and information in this article will highlight how social media can facilitate or hinder cyberbullying behaviors through anonymity in the online community to further assist intervention experts.

\section{Social Media in Context: Setting the Stage for Cyberbullying}

Social media is defined as, "Internet-based, disentrained, and persistent channels of masspersonal communication, facilitating perceptions of interactions among users, deriving value primarily from user-generated content" (Carr and Hayes 2015; p. 49). Social networking websites (Facebook, Google+, YouTube, Yelp, QQ, Pheed, etc.), social video games (Farmville, Second life, etc.), chat boards and blogs, professional networking websites (LinkedIn, Beehive, etc.), and socially designed mobile applications (Instagram, Yik Yak, etc.) are examples of social media platforms. Email correspondences, Skype conversations, online news services (e.g., CNN.com), and text message conversations are not considered social media platforms because of the lack of user-perceived interactions among a community of others that the user derives (Carr and Hayes 2015). Results from survey data collected in 2016 show that $69 \%$ of U.S. adults (aged 18-65 years) use at least one social media website and that rate increases to $86 \%$ when only 18-29-year-old adults are considered (Pew Research Center 2017). Furthermore, $92 \%$ of U.S. teens report going online daily, and $71 \%$ of those sampled use at least one social networking website (Lenhart 2015). Facebook is by far the most popular social media platform in both surveys.

Social media has evolved greatly since its inception. Earlier restricted and not user-friendly versions of social networking platforms existed in the 1970s and 1980s; however, the affordability of owning a PC in the home juxtaposed with the popularity of AOL in the mid-1990s jump-started the propensity for individuals to use social networking websites to communicate with others (DTS 2016). AOL allowed for individuals to create their own user name that could be searched through an online community to communicate digitally with others all over the world. The Internet boom of the early 2000s helped launch the popularity of various social networking websites, such as Friendster, MySpace, LinkedIn, Facebook, and other websites. The popularity of any specific social networking website waxed and waned with the changing face of the online landscape and the users of social media; however, social networking websites remained a popular staple for online communication. Most recently, the production of smart phones and tablets combined with easily accessible wireless Internet technology afforded users the ability to use social networking websites from their mobile devises (DTS 2016). Mobile phone applications were then created that allowed for users to post messages and communicate with others in an online community easily.

There are myriad reasons why individuals engage in social media communication. From a uses and gratifications theoretical framework perspective (a theory derived to explain why individuals consume specific media types; Rubin 1994), research has shown that social interactions, information seeking, passing the time, entertainment, relaxation, communicatory utility, and convenience are the primary motivations behind social media use (Dunne, Lawlor, and Rowley, 2010; Whiting and Williams 2013) —a finding largely replicated specific to Facebook (Park et al. 2009). Moreover, researchers (e.g., O'Keefe and Clarke-Pearson 2011) have noted many positive outcomes of social media use, including socialization (e.g., Bolton et al. 2013), enhanced learning in the classroom (e.g., Tess 2013), and accessing important health information (e.g., Moorhead et al. 2013).

Despite the potential positive outcomes associated with social networking use, additional research suggests that social media use is related to maladaptive behaviors, including cyberbullying (e.g., Whittaker and Kowalewski 2015). Individuals who want to inflict harm onto another online have many platform choices to cause their harm. The literature linking social media use and cyberbullying perpetration is under-researched and the paucity of research that has examined this relationship has yielded mixed results. Using primarily descriptive statistics, Whittaker and Kowalski (2015) reported that cyberbullying victimization among college-aged participants primarily happened through text messaging (56.8\%) followed by three social mediums: Twitter $(45.5 \%)$, Facebook (38.6\%), and Instagram (13.7\%). Mesch (2009) showed that having a social networking website (without detailing specifically which platforms) predicted online bullying in a sample of U.S. youth (aged 12-17 years), a finding replicated in a sample of German youth (aged 12-19 years; Festl and Quant 2013).

\section{Theoretical Considerations: Anonymous Cyberbullying}

The ability to theoretically link social media use to cyberbullying perpetration is important. If replicated and theoretically oriented empirical work can show why, when, and/or for whom social media use predicts cyberbullying perpetration, then interventions can be created or adapted to take these effects into account. However, popularity shifts of specific social media platforms juxtaposed with aesthetic and/or software changes have likely hindered such theoretical advances. For instance, in 2015, Whittaker and Kowalski showed that a small proportion of their sample (14.5\%) use instant messaging (IM) programs, which contrasted earlier work published in 2007, showing that $67 \%$ of college-aged participants often use IM websites (QuanHaase 2007). Moreover, Yik Yak (an online application wherein users affiliate themselves with a college community and post messages anonymously) decreased in popularity from 2014 (when it was the third most downloaded application) to 2016 (when it decreased to number 447 most popular application; McAlone 2016) likely, in part, due to software updates that required users to type in their phone number before commenting on or posting messages. 
Several broader social psychological theories have been applied to elucidate why individuals may engage in cyberbullying perpetration, such as the General Aggression Model (Anderson and Bushman 2002) and the Theory of Reasoned Action (Ajzen 2012). Unfortunately, none of these theories have explicated the link between social media use and cyberbullying perpetration and cannot reliably differentiate traditional (face-to-face) bullying from cyberbullying. We believe that social media frequency may be used to describe cyberbullying perpetration when situated in theory that is designed to uniquely predict cyberbullying by focusing on specific aspects of the social media platform: anonymity. The Barlett and Gentile Cyberbullying Model (BGCM; Barlett and Gentile 2012) theoretically elucidates the psychological mechanisms that predicts cyberbullying perpetration while simultaneously highlighting the differences between traditional bullying and cyberbullying. The BGCM posits that each online attack acts as a learning trial. As such, this theory suggests the aggressor will increasingly learn that two key factors in traditional bullying, identifiability and physical muscularity, are irrelevant when bullying on social media. Continued cyber attacks further reinforce these perceptions and beliefs until they become automatized, and form positive cyberbullying attitudes to predict cyberbullying perpetration. The BGCM is the only psychological model that incorporates and describes how anonymity perceptions predict cyberbullying behavior through the development of positive cyberbullying attitudes. Extensive research has supported the anonymity tenets of the BGCM using correlational (Barlett and Gentile 2012; Barlett 2015a) and longitudinal research designs (Barlett 2015b; Barlett et al. 2016; Barlett et al. 2017) on both college-aged samples (e.g., Barlett et al. 2017) and youth (e.g., Barlett 2015b; Wright 2013).

Our position is that, in accordance with BGCM postulates, social media platforms that allow for anonymous communication are more likely to facilitate cyberbullying perpetration than social media platforms that more likely identify the user. Indeed, Vandebosch and Van Cleemput (2008), Dooley and associates. (2009), and Thomas and colleagues. (2015) noted that the perception that an aggressor is more anonymous online is one factor that differentiates cyber from traditional bullying perpetration ${ }^{1}$. Although traditional bullies can aggress anonymously and research has shown that cyberbullies are also likely to be traditional bullies (e.g., Erdur-Baker 2010), Barlett (2015a) noted that the perception of anonymity online increases the likelihood of cyberbullying perpetration [see also Wright (2013)]. Patton and associates. (2014) noted that "Electronic technology also enables adolescents to hide their identities by sending or posting messages anonymously, by using a false name, or by assuming someone else's onscreen identity" (p. 550). In one study, Kowalski and Limber (2007) found in a survey of nearly 4000 youth that $48 \%$ of those who were cybervictimized were uncertain of the identity of the perpetrator. The Megan Meier, Ryan Halligan, Brandy Vela, and Amanda Todd stories that appear at the beginning of this article exemplify how social media can be used to

\footnotetext{
${ }^{1}$ These researchers also noted that the lack of physical contact, the meaning of the term repetition, and how power imbalance is construed also differentiate cyberbullying perpetration from traditional bullying perpetration.
}

deliver harmful online messages when the assailant perceives themselves to be anonymous.

Table 1 displays a list of some of the most popular social media platforms along with the year created, the number of users, whether the platform is accessible through computer, mobile phone, or both (termed medium), and information required to create an account. Examination of this table shows the variability in the amount of user information needed to create an account. Some platforms ask for very little personal information, which likely increases anonymity perceptions, whereas other platforms ask for several pieces of personal information. Independent of the amount of personal information, however, all social media platforms are prone to fake account creation. For instance, a user can make up a name and enter a newly created public email account to start communicating with others.

\section{Theoretical Considerations: Nonanonymous Cyberbullying}

Despite the research showing that anonymity perceptions predict cyberbullying attitudes (Barlett and Gentile 2012) and perpetration (Wright 2013), individuals may use social media platforms that are perceived by the user to be less anonymous to cyberbully others. In other words, we do not believe that perceived anonymity is neither a necessary nor sufficient condition to engage in cyberbullying. Whittaker and Kowalski (2015) used a program called Radian6 (software that can assess cyber-aggressive messages and trends using open-sourced nonpassword-protected social media) to statistically show that there were less directly aggressive online comments in Facebook (less likely anonymous) than a website's comment section (more likely anonymous); however, this effect was reversed when indirect aggressive online comments were analyzed. These findings suggest that the bluntness and clarity of harmful messages increase when users perceive themselves as anonymous, but aggressive online comments can still occur-although indirectly aggressive-when a user is more likely to be identified.

We are unaware of any research that has tested the motivations behind why people decide to engage or not engage in cyberbullying perpetration using anonymous or nonanonymous social media platforms. The BGCM is not yet able to address this issue; however, broader psychological theory can offer possible explanations. The online disinhibition effect (Suler 2004) posits that individuals may feel less restrained online (compared with face-to-face), which likely increases the likelihood of users expressing themselves more openly. Moreover, Suler differentiates benign disinhibition (where people share online kind generous sides of themselves or their emotions) from toxic disinhibition (where people engage in rude language, hatred, and threats, and show the more aggressive side of themselves) to suggest that not all disinhibited online behaviors are malevolent. Suler (2004) posits that perceived anonymity (creating fake names and accounts), invisibility of the user (the fact that two or more people cannot physically see each other online), asynchronicity (not interacting with people in real time), solipsistic introjection (reading online communication is experienced as if the sender is present in the mind), dissociative imagination (the ability to psychologically distance oneself from online happenings), and minimization of status and authority (the absence of 
Table 1. Descriptive Statistics for Popular Social Media Platforms

\begin{tabular}{llcll}
\hline Name & Medium & Year started & Number of users & \multicolumn{1}{c}{ Account creation information } \\
\hline Google+ & Both & 2011 & 111 Million & Valid email address (gmail) \\
Facebook & Both & 2004 & 2.01 Billion & Name and valid email address \\
Twitter & Both & 2006 & 328 Million & Name and valid email address \\
Qzone & Both & 2005 & 712 Million & Valid email address \\
Sina Weibo & Both & 2009 & 361 Million & Valid email address \\
Instagram & Both & 2010 & $700+$ Million & Name and valid email address \\
Tumblr & Both & 2007 & 367.2 Million & Name and valid email address \\
LinkedIn & Both & 2003 & $500+$ Million & Name and valid email address \\
Bebo & Both & 2005 & 117 Million & Name and valid email address \\
Tagged & Both & 2004 & 300 Million & Name, valid email address, and zip code \\
WhatsApp & Both & 2009 & 1 Billion & Name, valid email address, and a phone number \\
Messenger & Both & 2011 & 1.2 Billion & Name, valid email address, and a phone number \\
WeChat & Both & 2011 & 270 Million & Name, valid email address, and a phone number \\
Pinterest & Both & 2010 & 150 Million & Valid email address \\
Snapchat & Mobile & 2011 & 158 Million & Name, valid email address, date of birth, and a phone number \\
Vkontakte & Both & 2006 & 97 Million & Name and phone number \\
MySpace & Both & 2004 & 50 Million & Name and valid email address \\
Tinder & Mobile & 2012 & 50 Million & Name and valid email address \\
YouTube & Both & 2005 & 800 Million & Valid email address (gmail) \\
Yelp & Both & 2004 & 102 Million & Name and valid email address
\end{tabular}

Note that for the majority of these social media platforms, users can log in simply using their Facebook account credentials.

situational power or status cues in the online world) all contribute to online disinhibition. Research has shown that toxic online disinhibition is correlated with positive cyberbullying attitudes and perceived anonymity (Barlett and Helmstetter, in press), and cyberbullying perpetration (Barlett and Helmstetter, in press; Udris 2014).

\section{Social Media as a Tool to Reduce Cyberbullying}

\section{Reducing cyberbullying on social media: the issue}

Social media developers and companies are likely in a quandary regarding how to address cyberbullying perpetration and victimization. On one hand, the vast majority of social media platforms have code of conduct policies that specifies the consequences of harassment and bullying. For example, Facebook's community standards, which include an antiharassment policy, were redesigned in March 2015 such that their rules of conduct include (1) "Helping to keep you safe" that discusses the forbiddance of bullying and harassment, criminal activity, direct threats, among other negative interactions; (2) "Encouraging respectful behavior" that details the prohibition of hate speech, nudity, and other graphic content; (3) "Keeping your account and personal information secure" describes their policy on spam and fraud; and (4) "Protecting your intellectual property" that advocates users to post content from which they own the rights for. Facebook reserves the right to remove or delete accounts that violate these policies, which happened in the Brandy Vela example noted at the beginning of this article.

On the other hand, creating multiple fake social media accounts is not difficult on several social media platforms and it is likely impossible for a social media company to monitor every account to check for authenticity. For example, NBA superstar Kevin Durant reportedly has at least one "ghost" Twitter account that he uses to defend himself online from critics without people knowing it is actually him (Rosenberg
2017). The use of fake social media accounts is pervasive. It has been reported that 83 million Facebook accounts are either duplicate or fake accounts (Kelly 2012), and that 48 million Twitter accounts are not real people (Newberg 2017). Facebook reported that they recently deleted tens of thousands fake accounts before the 2017 election in Germany to help reduce the spread of "fake news" (Rogers 2017).

\section{Reducing cyberbullying on social media: possible solutions}

Fake social media accounts are easy to create and pervasive. We have argued that the anonymity perceptions likely generated from these accounts increase the likelihood that cyberbullying perpetration will be enacted. What can social media companies do to decrease the likelihood of cyberbullying beyond enforcing their code of conduct policies? We are not arguing that social media companies are solely responsible for reducing cyberbullying. The social media user, parental rules (if the user is a minor), antibullying school programs, personal ethics, and other entities should all concurrently work at addressing these issues. However, we do firmly believe that social media can help reduce cyberbullying perpetration. We offer three solutions that some social media companies already utilize.

First, social media sites should include settings that allow any user to combat cyberbullying. For example, filtering inappropriate language, blocking users, controlling who can see their social media activities, and reporting code of conduct violations are important. Many social media platforms have these suggestions in place. Results from a recent survey showed that (1) $62 \%$ of youth reported having their social media account set as a private profile compared with $17 \%$ who indicated having a public profile, (2) $59 \%$ of youth have deleted or edited something that they had posted before, $53 \%$ had deleted comments from others on their posts, and $45 \%$ had removed their name from photos that 
they had been tagged in, and (3) $31 \%$ had deactivated or deleted an entire account and $74 \%$ have deleted people from their network (Madden et al. 2013). Moreover, certain social media platforms (e.g., Twitter) have recently introduced new methods that allow users to report impersonations, self-harm, harassment on behalf of the users, or others. These statistics are encouraging and suggest that user-directed behaviors within social media sites allow for individuals to at least partially regulate their social media experience.

Second, social media platforms can attempt to encourage bystanders (individual who witness cyberattacks) to intervene. Research has shown a negative correlation between the number of bystanders and helping intentions in general situations (e.g., Darley and Latane 1968) and cyberbullying specific situations (Obermaier et al. 2016). Individuals online may be less likely to directly intervene; however, Dillon and Bushman (2015) had participants believe that they were piloting a new online chatting program before witnessing other "users" getting harassed online. Results showed that participants who noticed cyberbullying incidents were more likely to indirectly intervene (rate the cyberbully and the chatting program unfavorably) than directly intervene (direct correspondence related to cyberbullying to either the victim or cyberbully). Some social media platforms have methods for indirect intervention. For example, Yik Yak will have any message removed if enough users "down-vote" a specific post.

Third, the BGCM (Barlett and Gentile 2012) and past research (e.g., Barlett 2015a; Wright 2013) have shown that anonymity perceptions are related to cyberbullying-related outcomes. Social media platforms that are designed to ensure a nonanonymous experience should see a decrease in cyberbullying. For example, Twitter could force a user's real name to appear next to their online message rather than a usercreated ambiguous name. In addition, social networking websites could randomly send reauthentication checks to a user's registered email address, and if the email account is fake, then that account gets deactivated. Finally, social networking platforms could force users to type their phone number into the application before posting or commenting on a post, which should lead to a decrease in perceived anonymity and subsequent cyberbullying.

\section{Conclusion}

Social media usage has become an integral part of our world. Social media users can easily communicate with others all over the world. Despite the many positive uses of social media, some of these communication tools can be used to cause harm, and many youth are cybervictimized. Barlett $(2016,2017) \mathrm{ar}-$ gued that cyberbullying theory that highlights the differences between traditional and cyberbullying processes is needed to further understand cyberbullying perpetration in an effort to help inform interventions to reduce cyberbullying. However, there is limited theoretically driven empirical work testing the relationship between social media use and cyberbullying perpetration. By focusing on user-perceived anonymity while online, the BGCM offers one possible explanation regarding this relationship: if a social media platform allows the user to perceive themselves as anonymous - by the use of fake accounts, unidentifiable names or contact information, or bothcyberbullying through social media will persist. Additional research is desperately needed in this area. We sincerely hope that the present review will have a positive impact on the field by alerting researchers, theorists, intervention specialists, social media companies, school administrators, parents, and youth to the potential for online harm is likely increased by anonymous social media websites.

\section{Author Disclosure Statement}

No competing financial interests exist.

\section{References}

Ajzen I. (2012). The theory of planned behavior. In Handbook of Theories of Social Psychology. PM Van Lange, AW Kruglanski, E Higgins, eds. (Sage, London, UK), pp. 438-459.

Anderson CA, Bushman BJ. (2002). Human aggression. Annu Rev Psychol. 53, 27-51.

Barlett CP. (2015a). Anonymously hurting others online: The effect of anonymity on cyberbullying frequency. Psychol Popular Media Culture. 4, 70-79.

Barlett CP. (2015b). Predicting adolescent's cyberbullying behavior: A longitudinal risk analysis. J Adolesc. 41, 86-95.

Barlett CP. (2016). Past, present, and future theoretical developments in predicting cyberbullying behavior. In A Socio-Ecological Approach to Cyberbullying. M Wright, ed. (Nova Science Publishers, New York), pp. 13-28.

Barlett CP. (2017). From theory to practice: Cyberbullying theory and its application to intervention. Comput Hum Behav. 72, 269-275.

Barlett CP, Chamberlin K, Witkower Z. (2017). Predicting cyberbullying perpetration in emerging adults: A test of the Barlett Gentile Cyberbullying Model. Aggress Behav. 43, 147-154.

Barlett CP, Gentile DA. (2012). Attacking others online: The formation of cyber-bullying in late adolescence. Psychol Popular Media Culture. 1, 130-135.

Barlett CP, Gentile DA, Chew C. (2016). Predicting cyber-bullying from anonymity. Psychol Popular Media Culture. 5, 171-180.

Barlett CP, Helmstetter KM. Longitudinal relations between early online disinhibition and anonymity perceptions on later cyberbullying perpetration: A theoretical test on youth. Psychol Popular Media Culture. (in press).

Bolton RN, Parasuraman A, Hoefnagels A, et al. (2013). Understanding Generation $\mathrm{Y}$ and their use of social media: A review and research agenda. J Serv Manag. 24, 245-267.

Carr CT, Hayes RA. (2015). Social media: Defining, developing, and divining. Atlantic J Commun. 23, 46-65.

Darley JM, Latane B. (1968). Bystander intervention in emergencies: Diffusion of responsibility. J Person Soc Psychol. 8, 377-383.

Dillon KP, Bushman BJ. (2015). Unresponsive or un-noticed? Cyberbystander intervention in an experimental cyberbullying context. Comput Hum Behav. 45, 144-150.

Dooley JJ, Pyzalski J, Cross D. (2009). Cyberbullying versus face-toface bullying: A theoretical and conceptual review. J Psychol. 217, $182-188$.

DTS. (2016). The history of social networking. Retrieved from https:// www.digitaltrends.com/features/the-history-of-social-networking (accessed September 12, 2017).

Dunne A, Lawlor M, Rowley J. (2010). Young people's use of online social networking sites $-\mathrm{a}$ uses and gratifications perspective. Journal of Research in Interactive Marketing 4, 46-58.

Englander E, Donnerstein E, Kowalski R, et al. (2017). Defining cyberbullying. Pediatrics. 140(Suppl 2), S148-S151.

Erdur-Baker O. (2010). Cyberbullying and its correlation to traditional bullying, gender and frequent and risky usage of internet-mediated communication tools. New Media Soc. 12, 109-125. 
Ferguson JW. (2017). Long investigation leads to arrests in teen's suicide. Retrieved from www.galvnews.com/news/free/article_3cbd8fb0-a5265001-b9c6-2d4cfcd91751.html (accessed September 28, 2017).

Festl R, Quant T. (2013). Social relations and cyberbullying: The influence of individual and structural attributes on victimization and perpetration via the Internet. Hum Commun Res. 39, 101-126.

Kelly H. (2012). 83 million Facebook accounts are fakes or dupes. Retrieved from www.cnn.com/2012/08/02/tech/social-media/facebookfake-accounts (accessed September 28, 2017).

Kowalski RM, Giumetti GW, Schroeder AN, et al. (2014). Bullying in the digital age: A critical review and meta-analysis of cyberbullying research among youth. Psychol Bull. 140, 1073-1137.

Kowalski RM, Limber SP. (2007). Electronic bullying among middle school students. J Adolesc Health. 41, 22-30.

Lenhart A. (2015). Teens, social media, and technology: Overview 2015. Retrieved from www.pewinternet.org/2015/04/09/teenssocial-media-technology-2015 (accessed September 28, 2017).

Madden M, Lenhart A, Cortesi S, et al. (2013). Teens, social media, and privacy. Retrieved from www.pewinternet.org/2013/05/21/ teens-social-media-and-privacy (accessed September 22, 2017).

McAlone N. (2016). College students have totally lost interest in Yik Yak-And it could kill the app. Retrieved from www.businessinsider .com/yik-yak-losing-popularity-in-colleges-2016-9 (accessed September 12, 2017).

Menesini E, Nocentini A, Palladino BE, et al. (2012). Cyberbullying definition among adolescents: A comparison across six European countries. Cyberpsychol Behav Soc Netw. 15, 455-463.

Mesch GS. (2009). Parental mediation, online activities, and cyberbullying. Cyberpsychol Behav. 5, 387-393.

Microsoft. (2012). Online bullying among youth 8-17 years old: Worldwide. Retrieved from www.google.com/url?sa $=\mathrm{t} \& \mathrm{rct}=\mathrm{j} \& \mathrm{q}=$ \&esrc $=$ s\& $\&$ source $=$ web $\& c d=1 \&$ cad $=$ rja \&uact $=8 \& v e d=0 a h U K E w j X o$ YLLqaDWAhXoyFQKHfYxApkQFggoMAA\&url=http\%3A\%2F\%2 Fdownload.microsoft.com\%2Fdownload\%2Fe\%2F8\%2F4\%2Fe84bee ab-7b92-4cf8-b5c7-7cc20d92b4f9\%2Fww\%2520online\%2520bullying $\% 2520$ survey\%2520-\%2520 executive\%2520summary\%2520-\%2520 ww_final.pdf\&usg=AFQjCNG7Ncf0mc6BUDvJifk-FZ_cA1xdXQ (accessed September 12, 2017).

Moorhead SA, Hazlett DE, Harrison L, et al. (2013). A new dimension of health care: Systematic review of the uses, benefits, and limitations of social media for health communication. J Med Internet Res. 15, 1-26.

Newberg M. (2017). As many as 48 million Twitter accounts aren't people, says study. Retrieved from https://www.cnbc.com/2017/03/ 10/nearly-48-million-twitter-accounts-could-be-bots-says-study.html (accessed September 28, 2017).

Nobullying.org (2017). The top six unforgettable cyberbullying cases ever. Retrieved from https://nobullying.com/six-unforgettable-cyberbullying-cases (accessed September 28, 2017).

Obermaier M, Fawzi N, Koch T. (2016). Bystanding or standing by? How the number of bystanders affects the intention to intervene in cyberbullying. New Media Soc. 18, 1491-1507.

O'Keefe GS, Clarke-Pearson K. (2011). Clinical report-The impact of social media on children, adolescents, and families. Pediatrics. 127, 800-804.

Park N, Kee KF, Valenzuela S. (2009). Being immersed in social networking environment: Facebook groups, uses and gratifications, and social outcomes. Cyberpsychol Behav. 6, 729-733.
Patton DU, Hong JS, Ranney M, et al. (2014). Social media as a vector for youth violence: A review of the literature. Comput Hum Behav. $35,548-553$

PBS. (2008). Growing up online interviews: John Halligan. Retrieved from www.pbs.org/wgbh/pages/frontline/kidsonline/interviews/halligan .html (accessed September 28, 2017).

Pew Research Center. (2017). Social media fact sheet. Retrieved from www.pewinternet.org/fact-sheet/social-media (accessed September $28,2017)$.

Pokin S. (2007). Megan's story. Retrieved from https://www.meganmeier foundation.org/megans-story.html (accessed September 28, 2017).

Quan-Haase A. (2007). University students' local and distant social ties: Using and integrating modes of communication on campus. Inf Commun Soc. 10, 671-693.

Rogers J. (2017). Facebook deleted "tens of thousands" of fake accounts in the run-up to the German election. Retrieved from www.foxnews .com/tech/2017/09/27/facebook-deleted-tens-thousands-fake-accountsin-run-up-to-german-election.html (accessed September 28, 2017).

Rosenberg M. (2017). Kevin Durant's Twitter antics prove his critics right. Retrieved from https://www.si.com/nba/2017/09/18/kevindurant-twitter-fake-account-thunder-billy-donovan-russell-westbrook (accessed September 28, 2017).

Rubin AM. (1994). Media uses and effects: A uses and gratifications perspective. In Media Effects: Advances in Theory and Research. J Bryant, D Zillmann, eds. (Erlbaum Publishers, Hillsdale, NJ), pp. $417-436$.

Suler J. (2004). The online disinhibition effect. Cyberpsychol Behav. 7, 321-326.

Tess PA. (2013). The role of social media in higher education classes (real and virtual)—A literature review. Comput Hum Behav. 29, 60-68.

Thomas HJ, Cannor JP, Scott JG. (2015). Integrating traditional bullying and cyberbullying: Challenges of definition and measurement in adolescents: A review. Educ Psychol Rev. 27, 135-152.

Tokunaga RS. (2010). Following you home from school: A critical review and synthesis of research on cyberbullying victimization. Comput Hum Behav. 26, 277-287.

Udris R. (2014). Cyberbullying among high school students in Japan: Development and validation of the Online Disinhibition Scale. Comput Hum Behav. 41, 253-261.

Vandebosch H, Van Cleemput K. (2008). Defining cyberbullying: A qualitative research into the perceptions of youngsters. CyberPsychol Behav. 11, 499-503.

Whiting A, Williams D. (2013). Why people use social media: A uses and gratifications approach. Qual Market Res. 16, 362-369.

Whittaker E, Kowalski RM. (2015). Cyberbullying via social media. J Sch Violence. 14, 11-29.

Wright MF. (2013). The relationship between young adults' beliefs about anonymity and subsequent cyber aggression. Cyberpsychol Behav Soc Netw. 16, 858-862.

Address correspondence to: Christopher P. Barlett, PhD Department of Psychology Gettysburg College 300 N, Washington Street Gettysburg, PA 17325

E-mail: cbarlett@gettysburg.edu 